\title{
Sampled Medial Loci for 3D Shape Representation ${ }^{\text {is }}$
}

\author{
Svetlana Stolpner ${ }^{\mathrm{a}, *}$, Sue Whitesides ${ }^{\mathrm{b}}$, Kaleem Siddiqi $^{\mathrm{a}}$ \\ ${ }^{a}$ Centre for Intelligent Machines and School of Computer Science, McGill University, 3480 University \\ Street, Room 410, Montréal, Québec, Canada, H3A 2 A7 \\ ${ }^{b}$ Department of Computer Science, University of Victoria, PO Box 3055, STN CSC, Victoria, British \\ Columbia, Canada, V8W $3 P 6$
}

\begin{abstract}
The medial axis transform is valuable for shape representation as it is complete and captures part structure. However, its exact computation for arbitrary 3D models is not feasible. We introduce a novel algorithm to approximate the medial axis of a polyhedron with a dense set of medial points, with a guarantee that each medial point is within a specified tolerance from the medial axis. Given this discrete approximation to the medial axis, we use Damon's work on radial geometry [1] to design a numerical method that recovers surface curvature of the object boundary from the medial axis transform alone. We also show that the number of medial sheets comprising this representation may be significantly reduced without substantially compromising the quality of the reconstruction, to create a more useful part-based representation.
\end{abstract}

Keywords: medial representations, discrete differential geometry, medial simplification, 3D shape representation

\section{Introduction}

The ubiquity of high quality 3D polygonal mesh models in computer vision, computer graphics, medical imaging and solid modeling motivates the need for algorithms for the efficient and accurate analysis of the shape of these models. In this paper we

\footnotetext{
"This is an extended version of the article "Sampled Medial Loci and Boundary Differential Geometry" which appeared in the International Workshop on 3D Digital Imaging and Modeling, Kyoto, Japan, 2009.

* Corresponding author

Email address: sveta@cim.mcgill.ca (Svetlana Stolpner)
} 
consider a volumetric representation of such objects based on their medial axis transform [2]. This representation offers a powerful means by which to characterize both quantitative and qualitative aspects of 3D object geometry. However, the development of accurate and efficient algorithms for its computation remains a challenge and is an active area of research. Consider a solid $\Omega \subset \mathbf{R}^{3}$ with boundary $\mathcal{B}$.

Definition 1. A medial sphere of $\Omega$ is a maximal sphere inscribed in $\Omega$. A centre of a medial sphere is called a medial point.

A medial sphere has two or more points of tangency with the boundary $\mathcal{B}$. The directions from a medial point to its points of tangency with $\mathcal{B}$ have a special significance.

Definition 2. The vectors from a medial point $\mathbf{p}$ to any of its two closest points on $\mathcal{B}$ are the spoke vectors of $\mathbf{p}$.

These vectors are also called pannormals in the seminal work of Harry Blum [2].

Definition 3. The medial axis $\mathcal{M A}$ of $\Omega$ is the locus of centres of all medial spheres.

The medial axis we have thus defined is also known as the the interior 3D medial axis, or medial surface in the literature [3]. As an illustrative example, Figure 1 (left) depicts the medial axis of a box. It consists of several smooth surface patches, as is typical of the medial axis.

Definition 4. The medial axis transform of $\Omega$ is the set of all medial spheres of $\Omega$.

The medial axis transform is a valuable shape representation because it is complete, as shown in [2]:

Property 1 (Completeness). The union of the medial spheres of $\Omega$ is $\Omega$.

For a smooth object, the generic (i.e., stable under small perturbations of the object boundary) points of the $\mathcal{M A}$ fall into a small number of classes [4]. The majority lie on sheets (manifolds with boundary) of type $A_{1}^{2}$, i.e., they are points which have an order- 1 contact with the boundary at exactly 2 distinct points. These sheets intersect at curves of $A_{1}^{3}$ points and are bounded by curves of $A_{3}$ points (see Figure 1 (right)). A second important property of a medial representation is its ability to capture part structure, as shown in [4]: 
Property 2 (Part Structure). The medial axis generically has a natural part decomposition into medial sheets of $A_{1}^{2}$ points.

Objects that have tubular structure, i.e., ones that are circular in cross section, are nongeneric because their medial axis consists of curves, not sheets.

Applications of medial axis transform based representations of an object include object recognition and retrieval, object segmentation, object registration, statistical shape analysis, modeling shape deformations, path planning, as well as many others [3]. For these applications, it is often important to compute the medial axis transform in such a way that the above two properties, completeness and part structure, are captured.

In this article, we present the following algorithms and numerical methods:

1. An algorithm to obtain a dense set of points within a user-specified one-sided Hausdorff distance of the object's medial axis, along with their spoke vectors, in Section 3. The medial point cloud may be segmented into its constituent smooth sheets based on properties of the spoke vectors at each medial point.

2. A numerical method to estimate the principal curvatures and principal curvature directions at the two implied boundary patches on either side of each medial point, in Section 6. We show that our approximate, sampled medial representation contains sufficient information to qualitatively recover the principal curvatures of the boundary of the object it describes.

3. A method by which the individual sheets of the discrete medial axis approximation may be ordered by significance, in Section 7. This allows us to significantly reduce the size of the resulting part-based representation and to simplify the object shape.

The discrete medial axis representation computed with our method captures differential geometric properties of the object boundary, attesting to the completeness of this shape representation. Further, it allows for part structure to be recovered and for the parts to be ordered by significance.

This article is organized as follows. Sections 2 and 3 describe medial axis computation: Section 2 summarizes background and previous work, while Section 3 presents 
our algorithm for the approximation of the medial axis with points. Sections 4, 5, and 6 concern the evaluation of surface curvature: Sections 4 and 5 overview the mathematics of boundary curvature by evaluating either the boundary directly or using its medial axis transform, while Section 6 presents our numerical scheme for the estimation of differential quantities from sampled medial points. Section 7 concerns the simplification of our discrete approximation to the medial axis in terms of number of sheets.

\section{Medial Axis Computation}

For applications in computer vision and computer graphics, a 3D solid is often represented using a cloud of points sampled on its boundary or as a polyhedron. In this section, we first discuss some of the issues in the computation of the medial axis of 3D objects. Next, we survey some of the relevant methods that compute the medial axis of objects whose surface is represented as a closed polygonal mesh or as a cloud of points.

\subsection{Background}

When $\Omega$ is a polyhedron, its medial axis $\mathcal{M A}$ is composed of bisectors of the faces, edges and vertices of the polyhedron boundary $\mathcal{B}$. The bisector of two such elements is a quadric surface and these surfaces intersect along curves of higher algebraic degree. The exact medial axis of a polyhedron is difficult to compute exactly for models with a large number of faces. For higher-order boundary representations, the algebraic complexity of the medial sheets and their junctions increases.

For each pair of adjacent faces of a polyhedral boundary that meet convexly, the complete medial axis of the polyhedron contains points on the bisectors of these faces. This property results in a large number of medial sheets, but not all of these are deemed 'significant'. When approximating the medial axis, one often seeks to remove less significant portions in order to compute a simpler medial axis that continues to provide a nearly complete representation of the original object.

Two common measures of significance of a medial point include the radius of the associated medial sphere and the object angle of the medial point, defined as follows: 

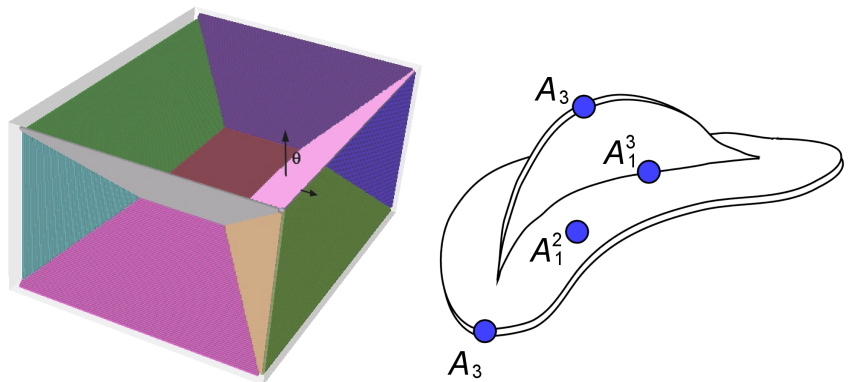

Figure 1: Left: The medial axis of a box, with each sheet shown in a different colour. The object angle $\theta$ at the selected medial point is $\pi / 4$. The spoke vectors are the black arrows. Right: Different classes of points that compose the medial locus of a smooth 3D object [4].

Definition 5. For an $A_{1}^{2}$ point $\mathbf{p}$, the object angle $\theta$ is the angle between the vector from $\mathbf{p}$ to either one of its two closest points on $\mathcal{B}$ and the tangent plane to $\mathcal{M A}$ at $\mathbf{p}$.

Figure 1 (left) shows a medial point with object angle $\theta$.

\subsection{Related Work}

The following methods compute the exact or approximate medial axis of a polyhedron. Culver et al. [5] use exact arithmetic to compute the seams of the medial axis of polyhedra having a small number of faces exactly. Etzion and Rappaport [6] perform octree spatial subdivision until each octree cell contains one vertex of the generalized Voronoi diagram of a polyhedron. Among the approximate approaches, Vleugels and Overmars [7] use octree spatial subdivision to approximate the medial axis of a set of disjoint convex polytopes in any dimension by considering the nearest boundary elements to the octree cell vertices. Foskey et al. [8] approximate the medial axis of a polyhedron with surface-aligned facets, with computations accelerated by graphics hardware on a voxel grid. Yang et al. [9] query points on spheres inside the solid and return midpoints of two neighbouring query points that have divergent directions to nearest boundary elements.

For 3D objects represented using a set of points sampled on their boundary, Voronoibased methods approximate their medial axis with a subset of the Voronoi vertices of the boundary points. Of these, [10] and [11] prove convergence of a subcomplex of 
the Voronoi diagram of the set of points sampled on the boundary of an object to the medial axis of the object as the sampling density approaches infinity. Leymarie and Kimia [12] compute a geometric directed hypergraph whose nodes are medial points, using exact bisector computations between clusters of surface points.

\section{Dense and Precise Medial Points}

We now describe a method that computes a set of sample points on the medial axis of a polyhedron with a user-specified density and precision. Based on the theoretical results in Section 3.1, we describe how the medial axis may be approximated with voxels in Section 3.2, how these estimates may be refined to provide a dense sampling of salient points near the medial axis in Section 3.3, how this point set may be segmented into sheets in Section 3.4, and show experimental results in Section 3.5.

\subsection{Foundations}

Our method is based on the analysis of the gradient of the Euclidean distance transform of our object $\Omega$ with boundary $\mathcal{B}$.

Definition 6. The Euclidean distance transform of a solid $\Omega$ with boundary $\mathcal{B}$ is given by $D(\mathbf{p})=\inf _{\mathbf{q} \in \mathcal{B}} d(\mathbf{p}, \mathbf{q})$, where $p \in \Omega$ and $d(\mathbf{p}, \mathbf{q})$ denotes Euclidean distance.

The gradient of $D, \nabla D: \mathbb{R}^{3} \rightarrow \mathbb{R}^{3}$ is hence a unit vector field where each point in the interior of $\Omega$ is assigned the direction to a (not necessarily unique) closest point $p$ on $\mathcal{B}$. This direction is the inward normal to $\mathcal{B}$ at $p$. The vector field $\nabla D$ is smooth everywhere except on the medial axis, where it is multi-valued: there are exactly 2 or more nearest boundary locations to points on the medial axis. This property is the basis for our method that locates medial points: we will look for regions where $\nabla D$ is multi-valued.

Consider a spherical region $S$ interior to $\mathcal{B}$ with outward normal $N_{S} \cdot{ }^{1}$ Then, as shown in $[13,14]$, in the continuum, the value of the average outward flux of $\nabla D$

\footnotetext{
${ }^{1}$ The choice of spherical region simplifies the analysis.
} 
through a shrinking region $S, \mathcal{A O F}_{S}(\nabla D)$, can be used to decide the presence and nature of the medial axis in $S$ as the area of $S$ tends to zero. Precisely,

$$
\mathcal{A O F}_{S}(\nabla D)=\frac{\iint_{S} \nabla D \cdot \mathbf{N}_{S} d S}{\iint_{S} d S} .
$$

If the medial axis does not intersect $S$,

$$
\lim _{\operatorname{area}(S) \rightarrow 0} \mathcal{A O F}_{S}(\nabla D)=0 .
$$

However, if $S$ contains a medial point with object angle $\theta$,

$$
\lim _{\operatorname{area}(S) \rightarrow 0} \mathcal{A O F}_{S}(\nabla D)=-\frac{1}{2} \sin (\theta) .
$$

It is important to note that this relationship holds only as the area of $S$ shrinks to zero.

We now describe how to use a similar measure to decide the presence of medial points in a non-zero size region $S$ when $\Omega$ is a polyhedron. In this special case, the $\nabla D$ vector field over the surface of a spherical region $S$ can be partitioned into a small number of classes. This is possible because the nearest locations on the boundary $\mathcal{B}$ of $\Omega$ to points $p$ inside $\Omega$ lie either (1) in the interior of a face, (2) in the interior of an edge, or (3) on a vertex of $\Omega$.

In the analysis of $\nabla D$ through $S$, it is important to define the notion of an opposite:

Definition 7. Consider a point p on the surface of a spherical region S. Let l be a line through a point $p$ with direction $\nabla D(p)$. Then the opposite of $p$, opp $(p)$ is the other point of intersection of $l$ with the surface of $S$ besides $p$.

Figure 2 shows examples of points and their opposites.

We define a special class of $\nabla D$ vectors for a boundary $\mathcal{B}$ :

Definition 8. $A$ two-sided fan for a $3 D$ solid with boundary $\mathcal{B}$ is a set of locations $p$ inside $\mathcal{B}$ such that $\nabla D($ opp $(p))=\nabla D(p)$ and $p$ lies on an edge or a vertex of $\mathcal{B}$.

The following theorem from [15] shows how an analysis of the average average flux through a region of non-zero size can be useful for detecting the medial axis of a polyhedron. 


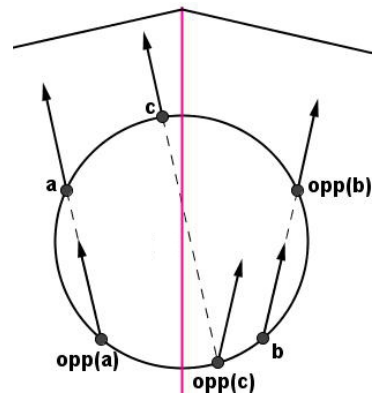

Figure 2: $\nabla D(a)=\nabla D(o p p(a))$ and $\nabla D(b)=\nabla D(o p p(b))$, while $\nabla D(c) \neq \nabla D(o p p(c))$. This means that for the boundary shown in black, the medial axis (in red) does not intersect the line segments $(a, o p p(a))$ and $(b, o p p(b))$, but does intersect the line segment $(c, o p p(c))$.

Theorem 1. Consider a spherical region $S$ inside a $3 D$ polyhedral solid $\Omega$ with boundary $\mathcal{B}$. Let $S^{*} \subset S$ be a set of locations on $S$ that are not two-sided fans for $\mathcal{B}$. The medial axis of $\Omega$ passes inside $S$ if and only if $\mathcal{A O F}_{S^{*}}(\nabla D)$ is negative.

The magnitude of $\mathcal{A O F}_{S^{*}}(\nabla D)$ remains proportional to the object angle of the medial axis that passes through $S$. In addition, it depends on the area of the intersection of the medial axis with $S$ : the magnitude of $\mathcal{A O F}_{S^{*}}(\nabla D)$ is smaller for smaller areas of intersection.

The integral in the numerator of Equation 1 is hard to compute exactly and it is desirable to approximate it. Using a simple quadrature rule, a discrete version of $\mathcal{A O F}_{S}(\nabla D)$ then is:

$$
A O F_{S}(\nabla D)=\frac{\sum_{i=1}^{m} \nabla D\left(\mathbf{p}_{i}\right) \cdot \mathbf{N}_{S}\left(\mathbf{p}_{i}\right)}{m}, p_{i} \in S .
$$

Unless the sampling rate of $S, m$, is infinite, only the forward implication of Theorem 1 holds. In other words, if $A O F_{S^{*}}(\nabla D)$ is negative, then necessarily the medial axis of $\Omega$ passes inside $S$. However, the medial axis may pass inside $\Omega$ even in the case that $A_{O F_{S^{*}}}(\nabla D)$ is non-negative for some finite value of $m$. This presents problems for the completeness of an algorithm that uses $A O F_{S^{*}}(\nabla D)$ to decide the presence of medial points. When $A O F_{S^{*}}(\nabla D)$ is non-negative, we are interested in analyzing the quality of potential medial points in $S$ as a function of their object angle, radius and $m$. For a $2 \mathrm{D}$ algorithm based on the analysis of $\nabla D$ samples, we have shown in [16] that, 
for any medial points not detected, either the object angle or radius decreases as more $\nabla D$ samples are considered in a region of interest. The success of the $2 \mathrm{D}$ analysis suggests that it is worth while to pursue a similar argument in 3D. However, the extension is not straightforward. We are actively researching worst-case theoretical bounds on the quality of our 3D algorithm.

In the following sections, we explain how the $A O F_{S^{*}}(\nabla D)$ measure can be used to efficiently locate a set of salient medial points of a polyhedron with a user-chosen density and precision.

\subsection{Approximating the Medial Axis with Voxels}

In this section we will use the foundations presented in the previous section to describe an algorithm to efficiently approximate the medial axis of $\Omega$ with voxels of a user-chosen size. The algorithm we will describe repeatedly evaluates the $A O F_{S^{*}}(\nabla D)$ measure for overlapping spherical regions interior to the object $\Omega$.

We overlay $\Omega$ with a coarse voxel grid where each voxel has side length $l$ and note those voxels such that a sphere $S$ of radius $l$ centred at the voxel center lies inside $\Omega$. We then repeat the following steps for each such voxel:

1. We distribute $m$ points approximately uniformly on the sphere $S$ centred at the voxel centre and with radius equal to the voxel length. We then compute $A O F_{S^{*}}(\nabla D)$, where $S^{*}$ is the subset of $S$ that does not contain any two-sided fans for $\nabla D$.

2. When this value is negative and we have reached the desired voxel resolution, we output this voxel. Otherwise, we subdivide the voxel considered into 8 equal size voxels and repeat steps 1-2 for the smaller voxels.

The result is a set of fine resolution voxels that are intersected by the medial axis of $\Omega$. We call this process of subdividing a voxel "zooming". Note that this coarse-to-fine procedure allows us to dismiss large regions that do not contain medial points early. In order to consider as much of the interior of $\Omega$ as possible, we repeat this procedure for any smaller resolution voxels near the boundary that were not interior to any coarselevel voxels. This step finds additional voxels that are intersected by the medial axis near the object boundary. 
To ensure efficiency of our algorithm, the computation of exact $\nabla D$ values, i.e., finding the nearest point on a mesh boundary to a query point, needs to be done quickly. To improve efficiently, we represent the boundary as a Sphere Swept Rectangle Hierarchy using the proximity query package PQP of [17]. This hierarchy allows to quickly find the nearest mesh triangle to a query point. The PQP package was designed to compute the exact shortest distance between pairs of triangle meshes (within machine precision). We have modified this package to work efficiently when one of the input objects is a point. As a heuristic, when "zooming", we save the nearest boundary triangles to points on the sphere bounding the top-level coarse voxel. When computing $\nabla D$ values for points inside this voxel, we then reduce the search for nearest mesh triangle to these saved triangles. When these saved triangles form a superset of nearest mesh triangles to points inside this voxel, the $\nabla D$ values are computed exactly (within machine precision).

\subsection{Precise, Salient Medial Points}

Given a set of voxels that are intersected by the medial axis, we would like to obtain more precise estimates of the location of the medial axis in these voxels. We will use the following property of the gradient of the Euclidean distance transform $\nabla D$, proved in [16].

Let $(\mathbf{a}, \mathbf{b})$ denote a line segment connecting points $\mathbf{a}$ and $\mathbf{b}$.

Lemma 1. Let $\mathbf{p}$ be a point in $\Omega$. Let $\mathbf{q}=\mathbf{p}+\gamma \cdot \nabla D(\mathbf{p})$, such that $(\mathbf{p}, \mathbf{q})$ lies inside $\Omega$ and $\gamma$ is a scalar. A medial point of $\Omega$ lies on $(\mathbf{p}, \mathbf{q})$ if and only if $\nabla D(\mathbf{p}) \neq \nabla D(\mathbf{q})$.

Using this lemma, we design subroutine RETRACT, shown as Algorithm 1, that performs binary search to estimate the intersection of the medial axis with a line segment to a desired accuracy.

By Lemma 1, Algorithm 1 finds a point on $(\mathbf{p}, \mathbf{q})$ that is within a user-specified tolerance $\epsilon$ of the medial axis $\mathcal{M A}$. Point $\mathbf{p}$ returned by Algorithm 1 is an approximate medial point. We consider the radius of the medial sphere at $\mathbf{p}$ to be the distance from $\mathbf{p}$ to the nearest location on $\mathcal{B}$ to $\mathbf{p}$. Note that, by definition, this sphere is interior and tangent to $\Omega$. 


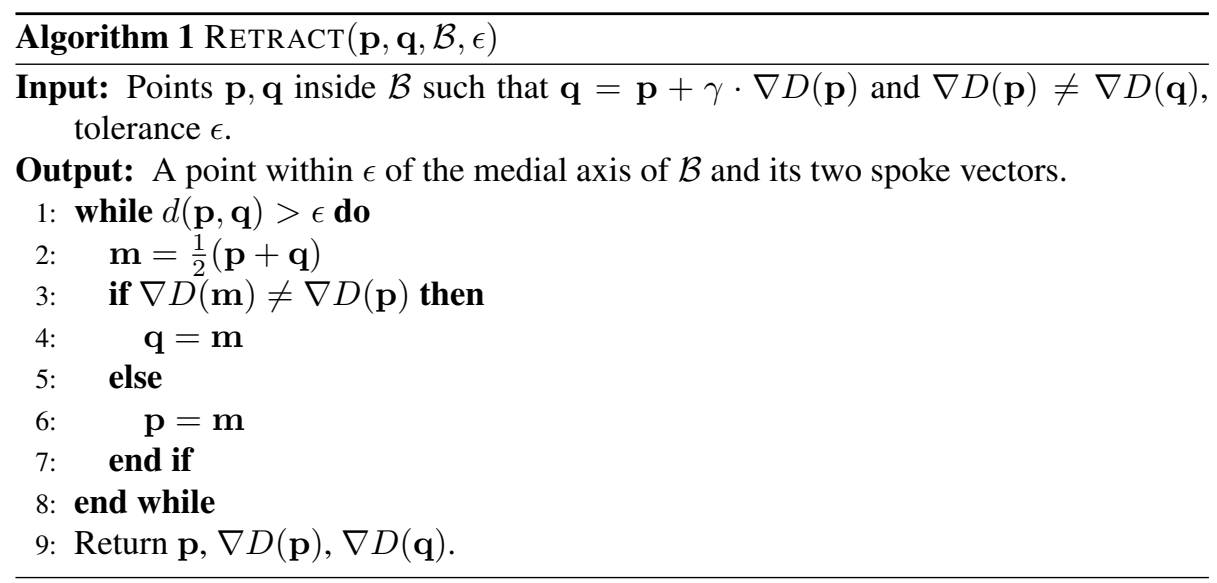

We wish to find a set of point samples that lies near a salient subset of the medial axis. We want the salient subset to have the property that the object may be accurately reconstructed from the computed medial spheres. A popular measure for the simplification of the medial axis is the object angle. Removal of medial spheres with a small object angle has been shown to have a small impact on the volume of the reconstructed object in [8]. However, simplification by object angle alone can disconnect an object. Chazal and Lieutier [18] prove that a simplification scheme based on the radius of the medial spheres preserves the topology of the medial axis. Attali et al. [19] argue in favour of a simplification scheme based on both object angle and radius. We adopt this strategy in our work in an effort to maintain the connectivity of the medial axis and achieve sufficient simplification.

For those voxels that are found to be intersected by the medial axis, Algorithm 2 looks for salient medial points inside this voxel and outputs one medial point per voxel if it is deemed salient in terms of the object angle and radius parameters.

Recall that the magnitude of $A O F_{S^{*}}(\nabla D)$ is proportional to the area of intersection of $\mathcal{M A}$ with $S$. Consider a voxel $v$ with side length $l$ circumscribed by a sphere with radius $l$. For efficiency reasons, if the object angle threshold is $\theta$, we ignore $v$ if the magnitude of $A O F_{S^{*}}(\nabla D)$ is below that given by a planar medial sheet with object angle $\theta$ intersecting $S$ in a circle with radius $\sqrt{3} l / 2$. This includes medial sheets that fall directly on a face of the voxel $v$ in $S$ and avoids missing sheets that pass on the 
border of 2 voxels. To avoid missing medial sheets with radii above the radius threshold $r$, we also consider those voxels for which the maximum distance to the mesh from sampled points exceeds $r-2 l$, where $l$ is the radius of the sphere placed around $v$.

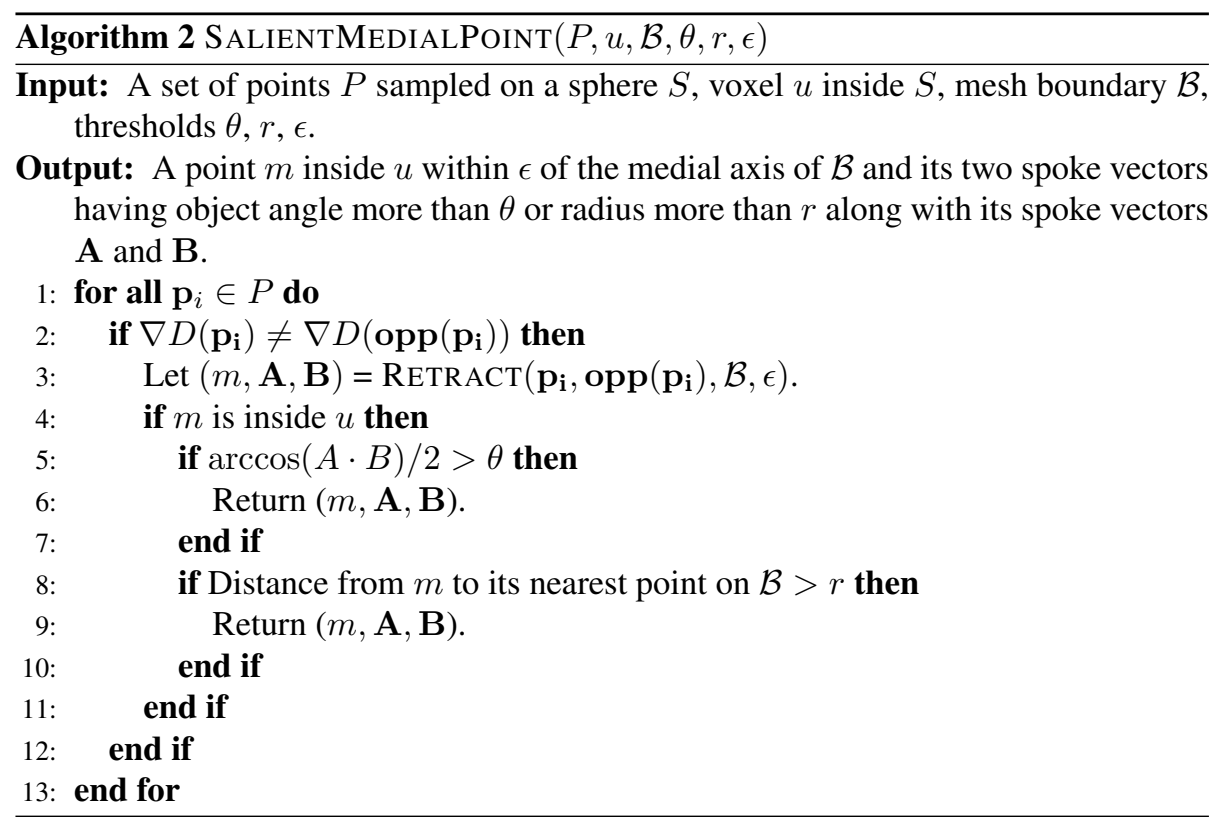

Using Algorithm 2, at most one salient approximate medial point is found per interior voxel. Thus, the density of the medial points is controlled by the resolution of the voxels considered.

\subsection{Medial Axis Segmentation}

Given a cloud of points sampled near the medial axis along with their estimated spoke vectors, we would like to segment the medial axis into its constituent sheets of $A_{1}^{2}$ points.

Given a sampling of $A_{1}^{2}$ points on a medial sheet, the normals to the medial sheet at these points do not always vary smoothly in a neighbourhood about a sampled point, as illustrated with the example in Figure 3. However, these sheets are smooth [20] and have the property that, for a pair of medial points $a$ and $b$ on such a sheet, there is a path on the sheet connecting $a$ and $b$, along which the normals to the medial sheet vary smoothly. We use this criterion to perform segmentation of the medial axis into sheets. 


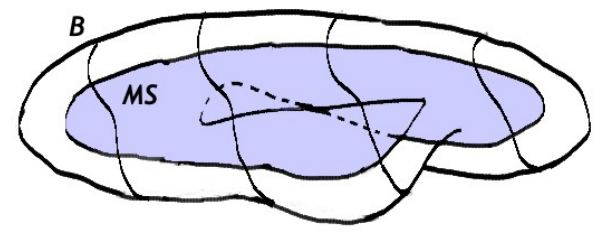

Figure 3: An example of an object with boundary $B$ whose medial axis is a single medial sheet $M S$ where neighbouring $A_{1}^{2}$ points have different surface normals. (Adopted from [20].)

To start, we need a notion of a surface normal to a medial point. It turns out that this information is encoded in our current representation.

Consider a medial point $\mathbf{x}_{0}$ and its two spoke vectors $\mathbf{U}_{\mathbf{x}_{0}}^{a}$ and $\mathbf{U}_{\mathbf{x}_{0}}^{b}$. The spoke vectors make an equal angle with the tangent plane to $\mathcal{M A}$ at $\mathbf{x}_{0}, T_{\mathbf{x}_{0}} \mathcal{M A}$. Let the normal to $T_{\mathbf{x}_{0}} \mathcal{M A}$ be $\mathbf{N}\left(\mathbf{x}_{0}\right)$. Then for $\mathbf{x} \in T_{\mathbf{x}_{0}} \mathcal{M} \mathcal{A}$,

$$
\begin{aligned}
& \mathbf{U}^{a}\left(\mathbf{x}_{0}\right) \cdot\left(\mathbf{x}_{0}-\mathbf{x}\right)=\mathbf{U}^{b}\left(\mathbf{x}_{0}\right) \cdot\left(\mathbf{x}_{0}-\mathbf{x}\right) \\
& \Rightarrow\left(\mathbf{U}^{a}\left(\mathbf{x}_{0}\right)-\mathbf{U}^{b}\left(\mathbf{x}_{0}\right)\right) \cdot\left(\mathbf{x}_{0}-\mathbf{x}\right)=0 .
\end{aligned}
$$

Therefore,

$$
\mathbf{N}\left(\mathbf{x}_{0}\right)=\mathbf{U}^{a}\left(\mathbf{x}_{0}\right)-\mathbf{U}^{b}\left(\mathbf{x}_{0}\right)
$$

is the normal direction to $T_{\mathbf{x}_{0}} \mathcal{M A}$.

We use these normal estimates to perform segmentation into sheets by grouping neighbouring medial points that have locally consistent normals. Specifically, for a medial point $\mathbf{q}$ on a sheet that is inside voxel $u_{\mathbf{q}}$, we add to this sheet all medial points $\mathbf{p}$ inside voxels in the 26-neighbourhood of $u_{\mathbf{q}}$ whose normals are within an allowed tolerance of the normal at $\mathbf{q}$. The result is a segmentation of the medial axis into smooth sheets of $A_{1}^{2}$ points.

\subsection{Experimental Results and Discussion}

Figure 4 (Centre) illustrates the union of the maximal spheres for the computed medial points with object angle threshold 0.3 radians and radius threshold 0.25 times the maximum dimension of the bounding box. These examples show that the union of the salient medial spheres provides a close approximation to the shape interior to 


\begin{tabular}{lcccc}
\hline Model & \# Triangles & $\begin{array}{c}\text { Final Voxel } \\
\text { Resolution }\end{array}$ & $\begin{array}{c}\text { \# Medial } \\
\text { Points }\end{array}$ & $\begin{array}{c}\text { Time } \\
(\mathrm{min})\end{array}$ \\
\hline Torus & 16,000 & $3,033,136$ & 64,195 & 5.93 \\
Pear & 86,016 & $5,177,526$ & 18,147 & 22.24 \\
Head & 6,816 & $5,244,401$ & 74,853 & 6.22 \\
Venus & 22,688 & $7,739,924$ & 116,793 & 12.82 \\
\hline
\end{tabular}

Table 1: Statistics for the computation of medial points.

the mesh boundaries. Figure 5 shows the importance of using the radius threshold in producing connected medial sheets.

Statistics for the computation of medial points for the models shown in Figure 4 are given in Table 1. The final voxel resolution is the number of finest-resolution voxels in the interior of the model. The number of medial points is the number of salient medial points found, at most one per finest-resolution voxel. Timings are shown for a single $3.4 \mathrm{GHz}$ Pentium IV processor with $3 \mathrm{~GB}$ of RAM. For practical purposes, using a smaller final voxel resolution and a coarser boundary mesh significantly speed up computation.

\section{Differential Geometric Shape Operator}

We have presented the medial axis transform as a volumetric shape representation for closed 3D objects. One of the natural ways to describe the shape of an object is to consider the amount of bending of its surface. The field of differential geometry investigates such measures. In this section, we first recall the basics of the differential geometric shape operator for objects with differentiable boundaries. Next, we explain how these measures have been estimated when the boundary is not differentiable, but approximated with a triangle mesh or a cloud of points. In Sections 5 and 6, we explain how these measures can be estimated from a discrete approximation to the medial axis transform.

Consider a surface $\mathcal{B} \subset \mathbb{R}^{3}$ with unit normal $\mathbf{n}$. For a point $\mathbf{x}_{0}$ on $\mathcal{B}$ and a tangent vector $\mathbf{v}$ to $\mathcal{B}$ at $\mathbf{x}_{0}$, define

$$
S(\mathbf{v})=-\frac{\partial \mathbf{n}}{\partial \mathbf{v}}
$$


Choosing an orthonormal basis $\left\{\mathbf{v}_{1}, \mathbf{v}_{2}\right\}$ for the tangent plane $T_{\mathbf{x}_{0}} B$ to $\mathcal{B}$ at $\mathbf{x}_{0}$,

$$
\frac{\partial \mathbf{n}}{\partial \mathbf{v}_{i}}=-a_{1 i} \cdot \mathbf{v}_{1}-a_{2 i} \cdot \mathbf{v}_{2}, i=1,2 .
$$

The matrix

$$
S=\left(\begin{array}{ll}
a_{11} & a_{12} \\
a_{21} & a_{22}
\end{array}\right)
$$

is the differential geometric shape operator. The eigenvalues of $S$ are the principal curvatures at $\mathbf{x}_{0}$ and the eigenvectors give the principal curvature directions. A good introductory text on differential geometry is [21].

For objects whose boundary is a non-differentiable, like a triangle mesh or a cloud of points, methods that estimate differential geometric quantities on the boundary may be divided into two classes: those that fit analytic functions to the data and those that work with the discrete data directly. In the former class, Cazals and Pouget [22] show that differential quantities evaluated for fitted polynomial surfaces of required degree converge to the true values given that a sampling condition on the boundary is met. In the latter class, Taubin [23] finds curvature tensors by considering estimates of normal curvature in a neighbourhood about each mesh vertex. The curvature values obtained from the curvature tensor in [24] approach the true values if a sampling condition on a particular kind of mesh is satisfied. Rusinkiewicz [25] performs this computation on a per-triangle basis. The work of Taubin [23] is extended to 3D range data in [26]. A survey of advances in this area can be found in [27].

\section{Boundary Geometry from Medial Geometry}

Since the medial axis transform is a complete shape descriptor, it is natural to ask if it is possible to estimate boundary curvature using this descriptor, without reconstructing the object boundary. To our knowledge, there has been little work that relates medial differential geometry to boundary geometry, with two exceptions. In [28] formulas are derived for the Gaussian and Mean curvatures for 3D boundaries based on derivatives along medial sheets, but this theory has not yet lead to implementations. For objects with non-branching medial topology, Yushkevich et al. [29] fit a single- 
sheet continuous medial representation (an $m$-rep) to medical image data and derive conditions to compute the implied boundary.

Recent work by differential geometer James Damon has rigorously established the relationship between medial and surface geometry. In this section, we overview some of the important results in [1] that will serve as the basis of our numerical method for the estimation of boundary differential geometry from a sampling of medial points in Section 6.

If $\mathcal{B} \subset \mathbb{R}^{3}$ is the boundary of an object $\Omega \subset \mathbb{R}^{3}$ with unit outward normal $\mathbf{n}$, then, as shown in the previous section, the rate of change of $\mathbf{n}$ along $\mathcal{B}$ describes the curvature of $\mathcal{B}$. Rather than studying the rate of change of $\mathbf{n}$ as one moves along $\mathcal{B}$, consider the rate of change of $\mathbf{n}$ as one moves along medial axis $\mathcal{M A}$ of $\Omega$. Recall that smooth $\left(A_{1}^{2}\right)$ medial points are equidistant from exactly 2 points on $\mathcal{B}$. The vectors from smooth medial points to nearest points on $\mathcal{B}$, the spoke vectors, are normal to $\mathcal{B}$. Thus, by studying the rate of change of the spoke vectors we obtain information about the rate of change of the normals to the boundary. This observation is the basis for the mathematics we develop in this section. We now proceed to define a special shape operator on the medial axis, Damon's radial shape operator [1].

Consider a smooth medial point $\mathbf{x}_{0} \in \mathcal{M A}$ and let $\mathbf{U}^{a}, \mathbf{U}^{b}$ be the 2 spoke vectors at $\mathbf{x}_{0}$. Let $\mathbf{U}_{1}^{a}=\mathbf{U}^{a} /\left\|\mathbf{U}^{a}\right\|, \mathbf{U}_{1}^{b}=\mathbf{U}^{b} /\left\|\mathbf{U}^{b}\right\|$. Damon defines

$$
\begin{aligned}
& S_{r a d}^{a}(\mathbf{v})=-\operatorname{proj}_{\mathbf{U}^{a}}\left(\frac{\partial \mathbf{U}_{1}^{a}}{\partial \mathbf{v}}\right) \text { and } \\
& S_{r a d}^{b}(\mathbf{v})=-\operatorname{proj}_{\mathbf{U}^{b}}\left(\frac{\partial \mathbf{U}_{1}^{b}}{\partial \mathbf{v}}\right),
\end{aligned}
$$

where $\operatorname{proj}_{\mathbf{U}}$ denotes projection into $T_{\mathbf{x}_{0}} \mathcal{M A}$ along $\mathbf{U}$ [1]. Refer to Figure 6.

Choosing an orthonormal basis $\left\{\mathbf{v}_{1}, \mathbf{v}_{2}\right\}$ for $T_{\mathbf{x}_{0}} \mathcal{M A}$, let $S_{\text {rad }}^{a}, S_{\text {rad }}^{b}$ denote the matrix representation of the two radial shape operators. Then, as in the case of the standard shape operator, the eigenvectors are principal radial curvature directions and the eigenvalues are principal radial curvatures. Let $\kappa_{r i}$ denote the principal radial curvatures. The following condition is required to ensure the smoothness of the boundary $\mathcal{B}$.

Definition 9. The Radial Curvature Condition is defined as follows. For all smooth 
medial points,

$$
r<\min \left\{\frac{1}{\kappa_{r i}}\right\}
$$

for all positive principal radial curvatures $\kappa_{r i}$.

Consider the correspondence between medial points and their closest boundary points given by $\psi^{a}: \mathcal{M A} \rightarrow B, \psi^{b}: \mathcal{M A} \rightarrow B$. Denote $\psi^{a}\left(\mathbf{x}_{0}\right)$ by $\mathbf{x}_{0}^{a}$ and $\psi^{b}\left(\mathbf{x}_{0}\right)$ by $\mathbf{x}_{0}^{b}$. It is shown in [1] that:

Theorem 1. Let $\mathbf{x}_{0}^{a}$ be a smooth medial point of an object with a smooth boundary $\mathcal{B}$ that satisfies the Radial Curvature Condition. The principal curvatures $\kappa_{i}^{a}$ of $\mathcal{B}$ at $\mathbf{x}_{0}^{a}$ and the principal radial curvatures $\kappa_{r i}$ of $\mathcal{M A}$ at a smooth medial point $\mathbf{x}_{0}$ have the following relationship:

$$
\kappa_{i}^{a}=\frac{\kappa_{r i}}{1-r \kappa_{r i}}
$$

where $r=\left\|\mathbf{U}^{a}\right\|$. Furthermore, the principal radial curvature directions corresponding to $\kappa_{r i}$ can be found by applying the map $\psi^{a}$ to the principal curvature directions corresponding to $\kappa_{i}^{a}$. The case of $S_{\text {rad }}^{b}$ is symmetric.

Thus, computing the radial shape operator at a smooth medial point of an object with a smooth boundary, one can find principal curvatures and principal curvature directions on the associated locations on the object boundary.

\section{Boundary Geometry from Sampled Medial Geometry}

In the same spirit as the body of work on adopting continuous mathematics for differentiable surfaces to surfaces represented by non-differentiable objects (see Section 4), we adopt the continuous formulations presented in the previous section to the situation where the smooth medial axis is approximated by a medial point cloud.

This point cloud is sampled near the medial axis, which is a connected set of manifolds with boundary. Reconstructing a smooth interpolating surface from this point set is a challenging open problem in the domain of surface reconstruction. Rather than performing computations on a surface that interpolates the medial points, we work with the set of medial points directly. 
In this section, we describe a numerical method by which boundary curvature may be inferred from a collection of medial points and their spoke vectors.

\subsection{Imposing Smoothness on the Boundary}

In our case, $\mathcal{B}$ is the boundary of a polyhedron and is piecewise planar. In order to satisfy the Radial Curvature Condition (Definition 9) at smooth medial points, we have to ensure that $r<\min \left\{\frac{1}{\kappa_{r i}}\right\}$ for positive values of $\kappa_{r i}$. Non-smooth objects

may not satisfy the Radial Curvature Condition. For such objects, $r \geq \min \left\{\frac{1}{\kappa_{r i}}\right\}$ for some medial points. When $r>\min \left\{\frac{1}{\kappa_{r i}}\right\}, \mathcal{B}$ locally self-intersects. When $r=$ $\min \left\{\frac{1}{\kappa_{r i}}\right\}, \mathcal{B}$ has a non-differentiable concavity, e.g., a concave edge or vertex for a polyhedron. When $\mathcal{B}$ is a non-convex polyhedron, the concave edges and vertices are likely to be nearest boundary points to medial points of the polyhedron. Figure 7 illustrates a scenario where two nearby medial points share the same nearest boundary point on a concave vertex. It can be easily verified that in this case, indeed, $r=\frac{1}{\kappa_{r i}}$. Therefore, the direct application of the above theory to non-convex piecewise planar boundaries is not valid.

For non-convex polyhedral shapes, we impose smoothness on the $\mathbf{U}^{a}$ and $\mathbf{U}^{b}$ vectors by performing a local Gaussian weighted average with neighbouring vectors. Specifically, the weights of the contribution of vectors at $\mathbf{x}_{1}$ to vectors at $\mathbf{x}_{0}$ are given by the Gaussian function $\frac{1}{(2 \pi)^{3 / 2} \sigma} e^{-\frac{\left\|\mathbf{x}_{0}-\mathbf{x}_{1}\right\|_{2}^{2}}{2 \sigma^{2}}}$, with $\sigma=0.25$. This corresponds to smoothing the boundary implied by the medial points and their spoke vectors. The use of this heuristic prohibits computing very large curvatures at reflex vertices of the mesh. We only perform this smoothing step for non-convex objects.

\subsection{Derivatives on Medial Sheets}

For a medial point $\mathbf{x}_{0}$, we now measure the rate of change of the spoke vectors $\mathbf{U}^{a}\left(\mathbf{x}_{\mathbf{0}}\right)$ and $\mathbf{U}^{b}\left(\mathbf{x}_{\mathbf{0}}\right)$ in the directions given by the basis $\left\{\mathbf{v}_{1}, \mathbf{v}_{2}\right\}$ to the tangent plane at $\mathrm{x}_{\mathbf{0}}, T_{\mathbf{x}_{0}} \mathcal{M A}$. To estimate an orthonormal basis to the tangent plane at $\mathbf{x}_{\mathbf{0}}$, we estimate the normal $\mathbf{N}_{\mathcal{M A}}\left(\mathbf{x}_{\mathbf{0}}\right)$ to the medial axis at $\mathbf{x}_{\mathbf{0}}$ using the method in Section 3.4 and find an orthonormal direction $\mathbf{v}_{1}$ to $\mathbf{N}_{\mathcal{M A}}\left(\mathbf{x}_{0}\right)$.

Given a particular step size $\Delta$ for the derivatives, we find another medial point $\mathbf{x}_{1}$ that is approximately distance $\Delta$ from $\mathbf{x}$ in the direction $\mathbf{v}_{1}$ and update the vector $\mathbf{v}_{1}$ 
to be the unit direction from $x_{0}$ to $x_{1}$. We consider the spoke vectors at $\mathbf{x}_{\mathbf{0}}$ and $\mathbf{x}_{\mathbf{1}}$. We disambiguate between the $\mathbf{U}^{a}$ and $\mathbf{U}^{b}$ vectors by computing consistent estimates of surface normals to the medial axis using the method of [11]. In order to ensure that $\mathbf{x}_{1}$ lies on the same medial sheet as $\mathbf{x}_{\mathbf{0}}$, we check that the normals to $\mathbf{x}_{1}$ and $\mathbf{x}_{\mathbf{0}}$ are similar.

For the $\mathbf{v}_{2}=\mathbf{N}_{\mathcal{M A}}\left(\mathbf{x}_{\mathbf{0}}\right) \times \mathbf{v}_{1}$ direction (recall that $\mathbf{v}_{1}, \mathbf{v}_{2}$ must give an orthonormal basis for the tangent plane at $\mathbf{x}_{0}$ ) we cannot expect to find a sampled point which lies exactly at $\mathbf{x}_{2}=\mathbf{x}_{\mathbf{0}}+\Delta \mathbf{v}_{2}$, because we are working with a discrete set of medial points. We therefore estimate $\mathbf{U}^{a}$ and $\mathbf{U}^{b}$ values at $\mathbf{x}_{2}$ as a Gaussian weighted average of the spoke vectors of the medial point samples in the vicinity of $\mathrm{x}_{2}$. Once again, to restrict computations to the same medial sheet, only those points in the vicinity that have similar normals to $\mathbf{N}_{\mathcal{M A}}\left(\mathbf{x}_{\mathbf{0}}\right)$ are considered.

At those medial points where this computation is defined, $\frac{\partial \mathbf{U}_{1}^{a}}{\partial \mathbf{v}}, \frac{\partial \mathbf{U}_{1}^{a}}{\partial \mathbf{v}}$ values are estimated for $\mathbf{v} \in\left\{\mathbf{v}_{1}, \mathbf{v}_{2}\right\}$ using forward differences.

\subsection{Computing Surface Curvature}

Given $\frac{\partial \mathbf{U}_{1}^{a}}{\partial \mathbf{v}}, \frac{\partial \mathbf{U}_{1}^{a}}{\partial \mathbf{v}}$ estimates for $\mathbf{v} \in\left\{\mathbf{v}_{1}, \mathbf{v}_{2}\right\}$, we now estimate the $S_{\text {rad }}$ operator at medial points and use its eigenvalues to find the principal curvatures $\kappa_{i}^{a}, \kappa_{i}^{b}, i=1,2$ at the two nearest points on the boundary $\mathcal{B}$ to the medial points.

In recovering the curvature of the original polygonal mesh boundary $\mathcal{B}$, we are presented with the obstacle that the set of nearest boundary points to the set of medial points we have computed does not include points on certain regions of $\mathcal{B}$. This happens because we retain salient medial points only. Hence, the geometry described by the medial points is that of the boundary of the union of our medial spheres, rather than that of the original boundary $\mathcal{B}$. For medial points that contribute a large spherical patch to the reconstruction of the shape, we only have curvature information about its 2 nearest boundary points (refer to Figure 8 for a 2D illustration). These points correspond to $A_{3}$ points in Giblin and Kimia's classification[4] (see Figure 1 (left)). Surface curvature values at missing locations are computed by propagating known surface curvature values along the boundary $\mathcal{B}$ to neighbouring surface points. 


\subsection{Experimental Results and Discussion}

We present numerical results on polyhedral objects of varying topology and surface complexity, and with varying medial axis branching topology: a three-hole torus, a pear, a head, and a Venus model. ${ }^{2}$ To show surface curvatures, we use the colormap in Figure 9 (bottom right). Here, red corresponds to a convexity, blue to a concavity, green to a saddle-shaped region, yellow to a cylindrical patch curving toward the object (the non-zero principal curvature is positive), cyan to a cylindrical patch curving away from the object (the non-zero principal curvature is negative) and white to a flat region. Figure 9 (left) shows the surface curvature estimates on the medial axis and Figure 9 (middle) shows these estimates projected onto the boundary $\mathcal{B}$. For comparison, Figure 9 (right) shows the result of applying Rusinkiewicz's method [25] directly on $\mathcal{B}$.

Although there are subtle numerical differences, the results obtained by the two methods are qualitatively very consistent. We emphasize that the implementation of our method works at a fixed spatial resolution on the set of medial points, whereas the method in [25] is aided by the explicit representation of the surface discontinuities of the mesh boundary and the connectivity of the mesh. The majority of surface regions recovered by our method are correctly coloured. Examples include the holes and the sides of the torus; the stem, neck and base of the pear; the eye sockets, neck, chin and nose of the head; and the neck, shoulders, breasts, chest, thighs, and navel of the Venus model.

In order to demonstrate the validity of the principal curvature direction estimates from medial geometry, Figure 10 illustrates these on a model of a cup. As explained in Section 5, these estimates are obtained from the eigenvectors of $S_{\text {rad }}$. Note how the directions are orthogonal and correspond to the directions of maximal bending (red) and minimal bending (blue), as one would expect.

There are certain challenges faced in the implementation of our method, including the difficulty of performing computations near edges of medial sheets ( $A_{3}$ curves), where the tangent plane cannot be accurately estimated, and on narrow sheets. Numer-

\footnotetext{
${ }^{2}$ Models from the Princeton Shape Benchmark, http://vcg.isti.cnr.it/polycubemaps/ models/, and http://www.cs.princeton.edu/gfx/proj/sugcon/models/
} 


\begin{tabular}{cccc}
\hline & Positive & Negative & Zero \\
\hline$\mu$ & 0.069834 & -0.087302 & $1.205 \times 10^{-6}$ \\
$\sigma$ & 0.000101 & 0.000148 & $8.707 \times 10^{-6}$ \\
\hline$\mu$ & 0.068380 & -0.087144 & $-8.973 \times 10^{-7}$ \\
$\sigma$ & 0.003784 & 0.002784 & $1.428 \times 10^{-5}$ \\
\hline
\end{tabular}

Table 2: Mean $(\mu)$ and standard deviation $(\sigma)$ of the 3 different types of curvature on a section of a cylindrical cup wall (shown in Figure 10) consisting of 551 vertices obtained using the method of [25] (Top) and our method (Bottom). We estimate the true positive curvature to be approximately 0.069898 and the true negative curvature to be approximately -0.0875045 .

ical errors can be mitigated, at least in part, by increasing the number of medial points (the voxel resolution) at the cost of increased computation time. We also note that the union of medial spheres, whose geometry the medial points describe, is a slightly different object than the original polyhedral object, as object angle simplification removes some small-scale boundary details. This fact should be taken into account when comparing the two different curvature estimates in Figure 9.

Since ground truth curvatures are not available for such complicated objects as those shown in Figure 9, it is not trivial to numerically evaluate the quality of our curvature estimates. However, for the simple example of a portion of a cylindrical cup wall, such that one side of the wall has positive and zero principal curvatures, while the other side has negative and zero principal curvatures, such a numerical comparison is feasible. Based on estimates of exterior and interior radii of the cup wall, we obtain an estimate for the positive and negative principal curvatures, respectively. The distribution of the curvatures estimated with our method and that of [25] is shown in Table 2. We observe that our relative error is $2.2 \%$ for the positive curvature and is $0.4 \%$ for the negative curvature.

Figure 11 presents results for another quantitative evaluation of our method. For the oblate spheroid considered, we compare the true principal curvatures to our principal curvature estimates. The finite difference approach to estimating the rate of change of the spoke vectors produces exact results when the spoke vector direction changes linearly over the neighbourhood, i.e., the boundary is round, like in the case of the cup model. For the spheroid, the boundary is not round and the error introduced by the approximation is expected to be larger. 
The estimation of boundary curvatures using this method was made possible due to the high density and precision of the medial point estimates. It is also very important to use a high-resolution boundary mesh. Having a large number of medial points with correct spoke estimates allows for finite differences of the spoke vectors in the tangent plane to the medial axis to capture relevant changes in surface normals.

\section{Medial Axis Simplification}

Being able to correctly compute a salient subset of the medial axis allows this representation to offer nearly complete information about the shape of the object being described. However, when working with natural shapes, the number of medial sheets computed can be large, despite simplification by object angle. In order for the sheets of the medial axis to be useful for a part-based analysis of the surface, their number should be manageable. It is also beneficial to assign each medial sheet a measure of significance. In this section, we propose a measure based on the volumetric contribution of each medial sheet to the union of medial spheres to order sheets by importance. Given a set of medial sheets salient in terms of object angle and radius, we find that often a small number of these sheets reconstructs the majority of the object volume.

\subsection{Related Work}

Several algorithms assign medial sheets a significance in order to either remove noisy, unstable sheets of the medial axis or to guide matching. When the goal is to simplify the medial axis while guaranteeing preservation of its homotopy type, methods in $[30,31,32]$ assign an ordering to the medial sheets that guides simplification. The thresholds for simplification are based on the object angle of the sheet [32] and the fraction of the volume of the object that would be removed as a result of pruning the sheet $[30,31]$. The significance assigned to a medial sheet by this volumetric measure depends on the ordering of the medial sheets considered.

Reference [33] considers the significance of a medial sheet, for the purpose of matching, to be the fraction of the total number of object voxels reconstructed uniquely by a medial sheet. Our approach is similar, but uses an exact volume measure that performs quickly in practice. 


\subsection{Unions of Spheres}

We approximate the medial axis transform of an object as a union of a finite number of its medial spheres. The union of spheres has been the subject of recent study in computational geometry. Below, we review some of the relevant results in this field that will be important for the formulation of a saliency measure for medial sheets.

Given a set of spheres, the space $\mathbb{R}^{3}$ may be partitioned into regions of influence of each sphere. The resulting space filling diagram, called a power diagram was introduced in [34] and is unlike the Voronoi diagram in that the radius of each sphere is considered in addition to the position of its centre. It is a valuable object for the computation of the volume of a union of spheres [35].

The power diagram is defined with respect to the power distance. The power distance between a point $x$ and a sphere $s=(c, r), d_{P}(x, s)$, is given as

$$
d_{P}(x, s)=d_{E}^{2}(x, c)-r^{2} .
$$

Then the power diagram is defined as:

Definition 10. Given a set of spheres, $S=\left\{s_{1}=\left(c_{1}, r_{1}\right), s_{2}=\left(c_{2}, r_{2}\right), \ldots, s_{n}=\right.$ $\left.\left(c_{n}, r_{n}\right)\right\}$, with centres $c_{i} \in \mathbb{R}^{3}$ and radii $r_{i} \in \mathbb{R}$, the power diagram of $S$, denoted $P D(S)$, is a partition of $\mathbb{R}^{3}$ into convex power cells $P\left(s_{i}\right)$, such that

$$
P\left(s_{i}\right)=\left\{x \in \mathbb{R}^{3} \mid d_{P}\left(x, s_{i}\right) \leq d_{P}\left(x, s_{j}\right), \forall j \neq i\right\} .
$$

Note that power cells are identical to Voronoi cells when all the sphere radii are the same. Figure 12 shows a 2D example of the power diagram of a set of disks.

The union of spheres may be decomposed by intersecting each sphere with its convex power cell.

Consider the dual $D C(S)$ of this decomposition, which is a simplicial complex that captures the topology of $S . D C(S)$ contains a vertex $i$ for each sphere $s_{i}$ and an edge $(i, j)$ whenever spheres $s_{i}$ and $s_{j}$ share a face of $P D(S)$. For a given sphere $s_{i}$, its power cell can be computed as follows. For each edge $(i, j)$ in $D C(S)$, we define a plane that is equidistant in power distance from spheres $s_{i}$ and $s_{j}$. For each such plane, consider a half-space that contains $s_{i}$. Then the power cell $P\left(s_{i}\right)$ of $s_{i}$ is the intersection of these half-spaces. 
It follows that the exact volume of a given union of spheres $S, \operatorname{vol}(S)$, can be computed by summing the contributions of each sphere restricted to its power cell:

$$
\operatorname{vol}\left(\cup_{i} s_{i}\right)=\sum_{i} \operatorname{vol}\left(s_{i} \mid P\left(s_{i}\right)\right)
$$

\subsection{Significance Measure}

A measure of significance of a medial sheet should reflect the role the sheet plays in the representation of the shape with respect to the other sheets. Also, this measure should be independent of the order in which sheets are processed. We develop such a measure based on the volume contribution of a medial sheet to the medial axis. Since our object $\Omega$ is approximated as a union of medial spheres, we use tools developed above to compute the significance of each medial sphere.

Let $S$ be the set of medial spheres of an object $\Omega$ whose union approximates $\Omega$. For a sphere $s_{i} \in S$, let $P\left(s_{i}\right)$ denote the power cell of $s_{i}$ in the power diagram of $S$. Then the significance of sphere $s_{i}$ to $S, \lambda\left(s_{i}\right)$, is given by the volume contribution of $s_{i}$ restricted to its power cell, relative to the total volume of the union of spheres:

$$
\lambda\left(s_{i}\right)=\frac{\operatorname{vol}\left(s_{i} \mid P\left(s_{i}\right)\right)}{\operatorname{vol}(S)} .
$$

Let $T \subseteq S$ be a set of medial spheres whose centres lie on the same medial sheet. The significance of $T=\left\{t_{i} \in S, i=1, \ldots,|T|\right\}, \lambda(T)$, is given by

$$
\lambda(T)=\sum_{i=0}^{|T|} \lambda\left(t_{i}\right) .
$$

\subsection{Experimental Results and Discussion}

Given this significance measure, we consider the subsets of spheres in $S$ belonging to separate medial sheets in descending order by significance. As more sheets are considered for the approximation of the shape, we can compute the degree to which $S^{\prime} \subset S$ approximates $S$ by evaluating $\operatorname{vol}\left(S^{\prime}\right) / \operatorname{vol}(S)$. Figures 13 and 14 show an ordering of the medial sheets of a pear and a head model. We observe that it is possible to reconstruct the majority of the volume of the original union of spheres using a small fraction of the original medial sheets. 
The computation of the volume of a sphere in a union of spheres restricted to its power cell is performed very quickly using the highly accurate software AlphaBall. The software takes approximately 5 seconds to compute this quantity for 20,000 spheres for a single $3.6 \mathrm{GHz}$ Pentium IV processor with $3 \mathrm{~GB}$ of RAM.

Because the medial axis usually consists of a large number of sheets, the use of the full medial axis is challenging for applications that consider the individual medial sheets, such as shape segmentation, animating deformations and shape matching. Our approach to simplifying the medial representation takes advantage of the combinatorial structure of a union of spheres to produce a more manageable representation in terms of the number of parts.

\section{Conclusion}

We have described a method to compute a dense collection of medial points, along with their spoke vectors, near the salient portions of the medial axis of a polyhedron. This discrete approximation to the medial axis transform is shown to be sufficient to recover qualitatively consistent surface curvatures on the object boundary. By decomposing the union of medial spheres into regions of influence of each sphere, we are able to compute the individual contribution of each medial sphere to the union. This measure is used to order medial sheets by significance and then to reduce the number of medial sheets required to approximate a shape. Thus, our discrete medial representation is a valuable approximation to the medial axis transform as it carries two desirable properties - ability to accurately describe the shape of the object represented, and ability to partition the object into a small number of volumetrically salient parts.

\section{Acknowledgements}

We thank the reviewers for their helpful comments. This research was funded by NSERC Canada and FQRNT Québec. We are grateful to Patrice Koehl for sharing with us the AlphaBall software. We are grateful to Szymon Rusinkiewicz for making the software package trimesh 2 publically available. We thank Tamal Dey for the NormFet software. 


\section{References}

[1] J. Damon, Determining the geometry of boundaries of objects from medial data, International Journal of Computer Vision 63 (1) (2005) 45-64.

[2] H. Blum, Biological Shape and Visual Science, Journal of Theoretical Biology 38 (1973) 205-287.

[3] K. Siddiqi, S. Pizer (Eds.), Medial representations: mathematics, algorithms and applications, Springer, 2008.

[4] P. J. Giblin, B. B. Kimia, A formal classification of 3D medial axis points and their local geometry, IEEE Transactions on Pattern Analalysis and Machine Intelligence 26 (2) (2004) 238-251.

[5] T. Culver, J. Keyser, D. Manocha, Exact computation of the medial axis of a polyhedron, Computer Aided Geometric Design 21 (1) (2004) 65-98.

[6] M. Etzion, A. Rappoport, Computing voronoi skeletons of a 3-d polyhedron by space subdivision, Computational Geometry: Theory and Applications 21 (2002) $87-120$.

[7] J. M. Vleugels, M. H. Overmars, Approximating generalized voronoi diagrams in any dimension, Tech. Rep. UU-CS-1995-14, Department of Information and Computing Sciences, Utrecht University (1995).

[8] M. Foskey, M. C. Lin, D. Manocha, Efficient computation of a simplified medial axis, in: Solid modeling and applications, 2003, pp. 96-107.

[9] Y. Yang, O. Brock, R. N. Moll, Efficient and robust computation of an approximated medial axis, in: Solid modeling and applications, 2004, pp. 15-24.

[10] N. Amenta, S. Choi, R. Kolluri, The Power Crust, Unions of Balls, and the Medial Axis Transform, Computational Geometry: Theory and Applications 19 (2-3) (2001) 127-153. 
[11] T. K. Dey, J. Sun, Normal and feature approximations from noisy point clouds, in: Foundations of Software Technology and Theoretical Computer Science, 2006, pp. 21-32.

[12] F. F. Leymarie, B. B. Kimia, The medial scaffold of 3d unorganized point clouds, IEEE Transactions on Pattern Analalysis and Machine Intelligence 29 (2) (2007) 313-330.

[13] K. Siddiqi, S. Bouix, A. R. Tannenbaum, S. W. Zucker, Hamilton-Jacobi skeletons, International Journal of Computer Vision 48 (3) (2002) 215-231.

[14] P. Dimitrov, J. N. Damon, K. Siddiqi, Flux invariants for shape, in: CVPR, 2003, pp. 835-841.

[15] S. Stolpner, K. Siddiqi, Revealing significant medial structure in polyhedral meshes, in: 3D Data Processing, Visualization, and Transmission, 2006, pp. 365372.

[16] S. Stolpner, S. Whitesides, Medial axis approximation with bounded error, in: International Symposium on Voronoi Diagrams, 2009, pp. 171-180.

[17] E. Larsen, S. Gottschalk, M. C. Lin, D. Manocha, Fast proximity queries with swept sphere volumes, Tech. rep., University of North Carolina at Chapel Hill (1999).

[18] F. Chazal, A. Lieutier, The $\lambda$-medial axis, Graphical Models 67 (4) (2005) 304331.

[19] D. Attali, J.-D. Boissonnat, H. Edelsbrunner, Stability and computation of the medial axis - a state-of-the-art report, in: Mathematical Foundations of Scientific Visualization, Computer Graphics, and Massive Data Exploration, SpringerVerlag, 2007.

[20] J. Damon, Tree structure for contractible regions in $\mathbb{R}^{3}$, Int. J. Comput. Vision 74 (2) (2007) 103-116. 
[21] B. O'Neill, Elementary Differential Geometry, 2nd Edition, Academic Press, 1997.

[22] F. Cazals, M. Pouget, Estimating differential quantities using polynomial fitting of osculating jets, in: Symposium on Geometry processing, 2003, pp. 177-187.

[23] G. Taubin, Estimating the tensor of curvature of a surface from a polyhedral approximation, in: International Conference on Computer Vision, 1995, p. 902.

[24] D. Cohen-Steiner, J.-M. Morvan, Restricted delaunay triangulations and normal cycle, in: Symposium on Computational geometry, 2003, pp. 312-321.

[25] S. Rusinkiewicz, Estimating curvatures and their derivatives on triangle meshes, in: 3D Data Processing, Visualization, and Transmission, 2004, pp. 486-493.

[26] E. Hameiri, I. Shimshoni, Estimating the principal curvatures and the darboux frame from real 3d range data., in: 3D Data Processing, Visualization, and Transmission, IEEE Computer Society, 2002, pp. 258-267.

[27] S. Petitjean, A survey of methods for recovering quadrics in triangle meshes, ACM Computing Surveys 34 (2) (2002) 211-262.

[28] L. R. Nackman, S. Pizer, Three dimensional shape description using the symmetric axis transform I: Theory, IEEE Transactions on Pattern Analalysis and Machine Intelligence 7 (2) (1985) 187-202.

[29] P. A. Yushkevich, H. Zhang, J. C. Gee, Continuous medial representation for anatomical structures., Transactions on Medical Imaging 25 (12) (2006) 154764.

[30] M. Styner, G. Gerig, S. C. Joshi, S. M. Pizer, Automatic and robust computation of $3 \mathrm{~d}$ medial models incorporating object variability, International Journal of Computer Vision 55 (2-3) (2003) 107-122.

[31] R. Tam, W. Heidrich, Shape simplification based on the medial axis transform, in: VIS '03: Proceedings of the 14th IEEE Visualization 2003 (VIS'03), Washington, DC, USA, 2003, p. 63. 
[32] A. Sud, M. Foskey, D. Manocha, Homotopy-preserving medial axis simplification, in: SPM '05: Proceedings of the 2005 ACM symposium on Solid and physical modeling, 2005, pp. 39-50.

[33] K. Siddiqi, J. Zhang, D. Macrini, A. Shokoufandeh, S. Bouix, S. J. Dickinson, Retrieving articulated 3D models using medial surfaces, Machine Vision and Applications 19 (4) (2008) 261-274.

[34] F. Aurenhammer, Power diagrams: properties, algorithms and applications, SIAM J. Comput. 16 (1) (1987) 78-96.

[35] H. Edelsbrunner, The union of balls and its dual shape, in: SCG '93: Proceedings of the ninth annual symposium on Computational geometry, 1993, pp. 218-231. 

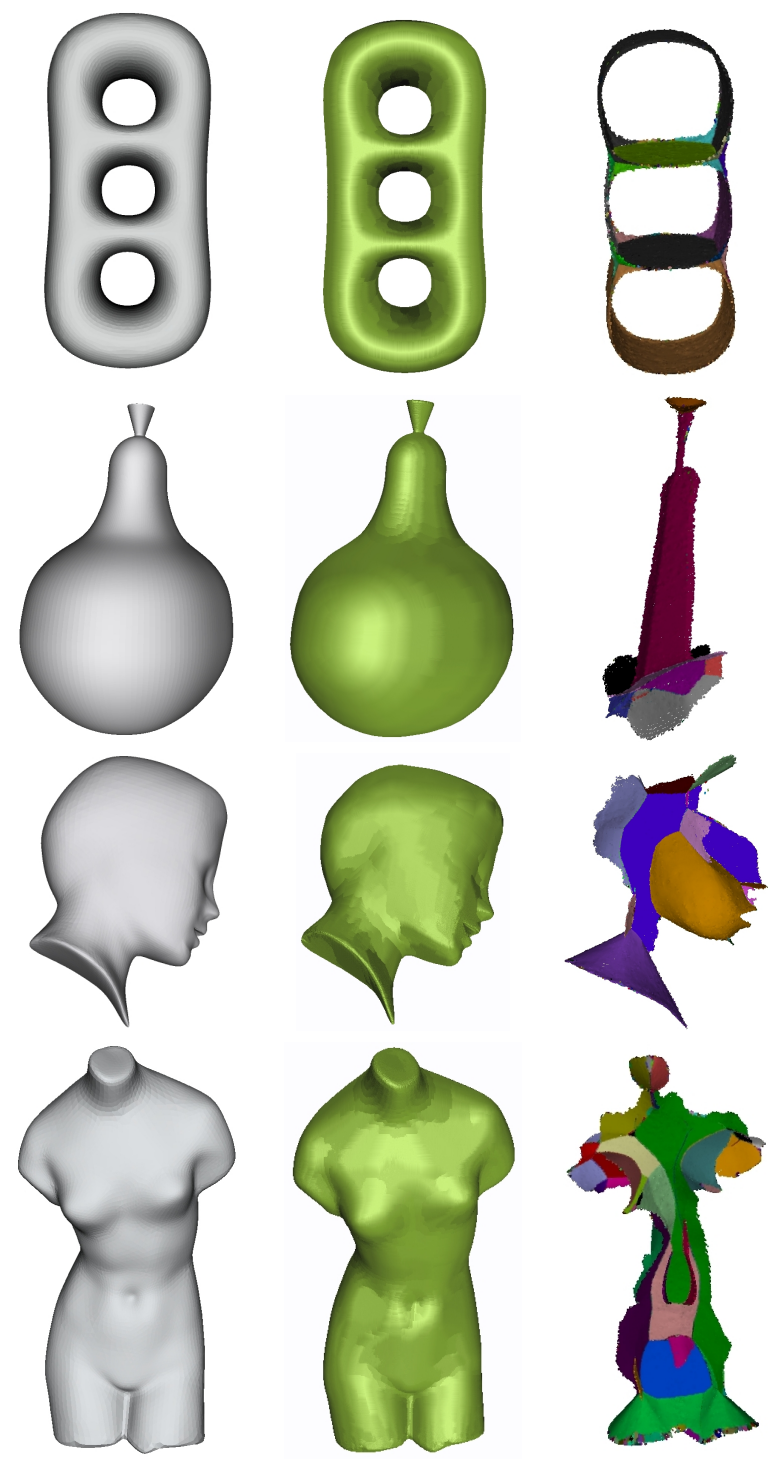

Figure 4: Left: The original polyhedra; Centre: the union of medial spheres; Right: medial points grouped into smooth sheets. The allowed tolerance between adjacent normals is 5.7 degrees. 

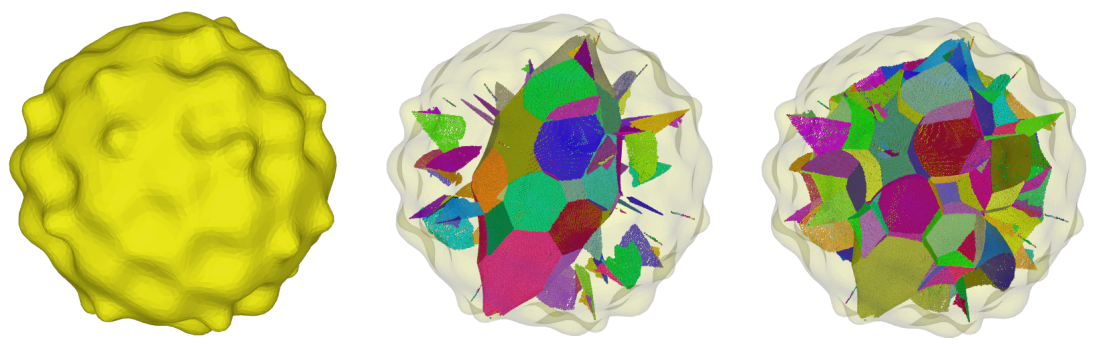

Figure 5: Left: A bumpy sphere model; Centre: Its medial axis when $\theta=0.6$ and no radius threshold is used is disconnected; Right: The medial axis when $\theta=0.6$ and a radius threshold of $10 \%$ of the maximum dimension of the bounding box is used.

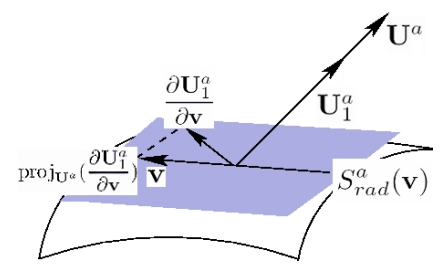

Figure 6: An illustration of $S_{r a d}^{a}(\mathbf{v})$ for a given $\mathbf{U}^{a}$ (adapted from [1]).

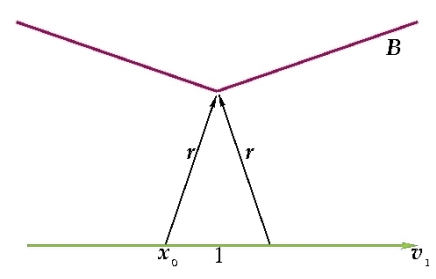

Figure 7: The case when $\kappa_{r i}=-\operatorname{proj}_{\mathbf{U}} \frac{\partial \mathbf{U}_{1}}{\partial \mathbf{v}_{1}}=\frac{1}{r}$. 


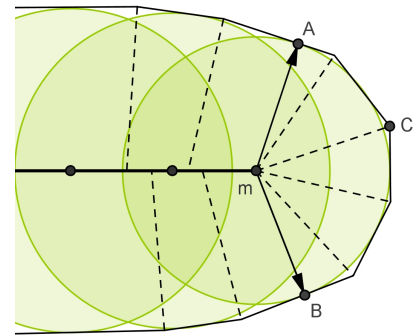

Figure 8: The medial axis of this object consists of low-object angle segments (dashed lines) and a highobject angle segment (bold line). When approximating the medial axis with a set of points, we only retain points on high-object angle segments of the medial axis. The boundary of the union of the associated medial circles approximates the original object. Medial point $m$ is equidistant from points $A$ and $B$ on the boundary. Surface curvatures at points $A$ and $B$ are found using the radial shape operator, while for point $C$ a different strategy is used. 

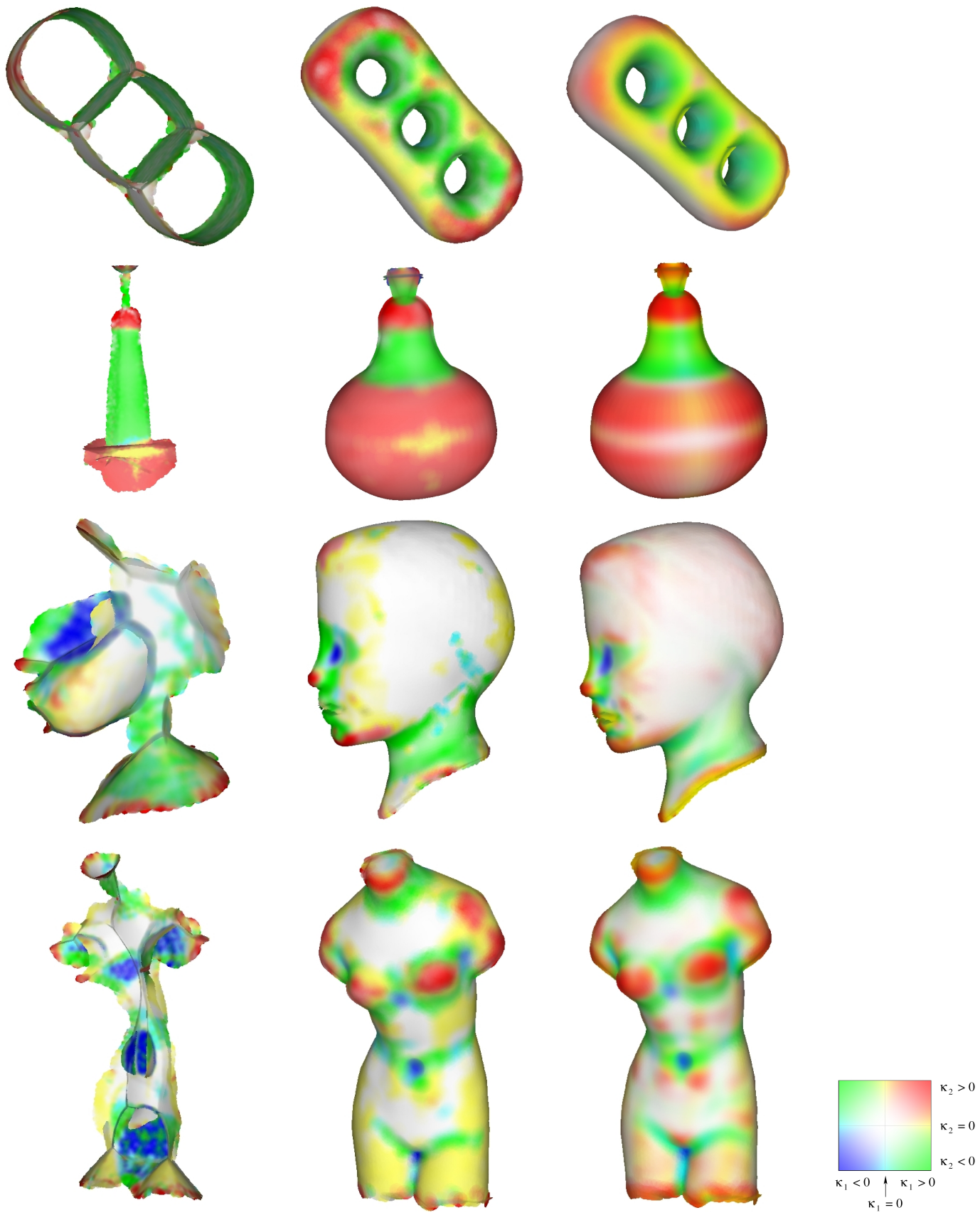

Figure 9: Left: The medial axis coloured according to principal curvature on the object boundary. Centre: Projection of curvature values on the medial axis to the boundary of the object. Right: Curvature values obtained using the method in [25]. The colourmap used is shown in the bottom right corner. See the associated text for a discussion of these results. 


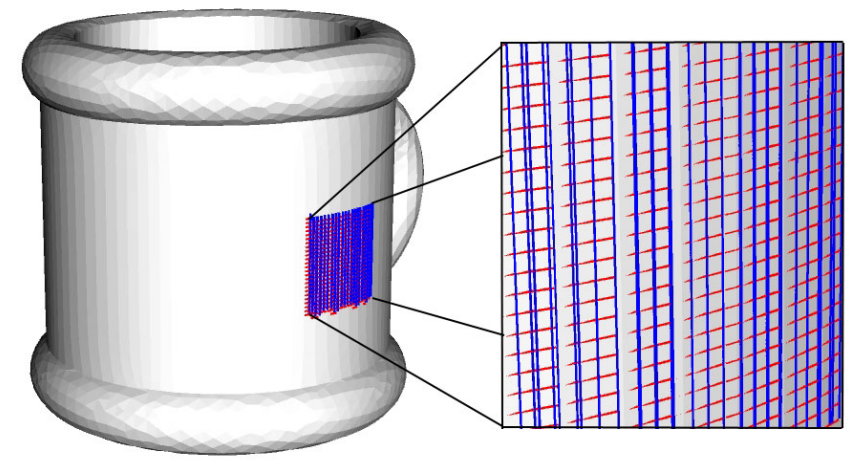

Figure 10: The principal curvature directions recovered on the surface of a cup using only the medial geometry. 


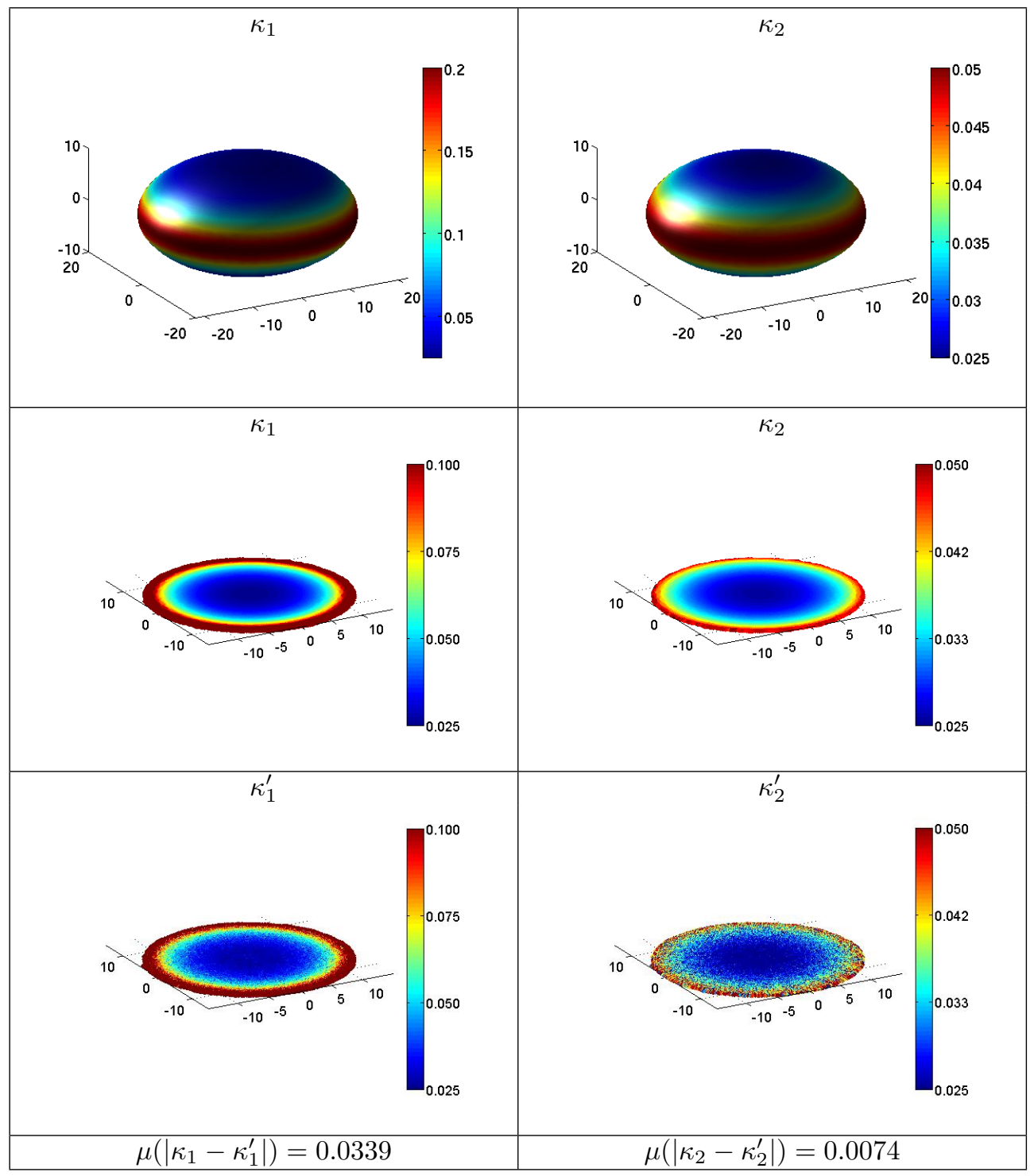

Figure 11: Top: An oblate spheroid coloured by principal boundary curvature $\kappa_{1}$ (left) and $\kappa_{2}$ (right). Middle: The medial axis of the oblate spheroid coloured by principal boundary curvature. Bottom: Respective principal curvature estimates $\kappa_{1}^{\prime}$ and $\kappa_{2}^{\prime}$ shown on the medial axis. The mean absolute error of the estimation of $\kappa_{1}$ and $\kappa_{2}$ is shown below each column. Please refer to the online version of this article for the colour version of this and other figures. 


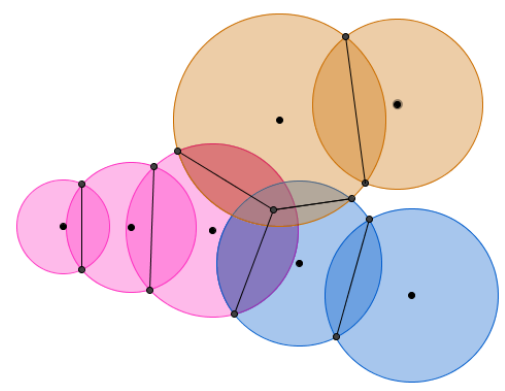

(a)

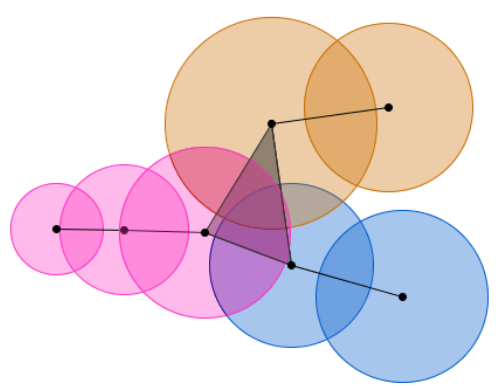

(b)

Figure 12: (a) A union of the set of disks decomposed using the power diagram; (b) the dual of the decomposition of the set of disks.

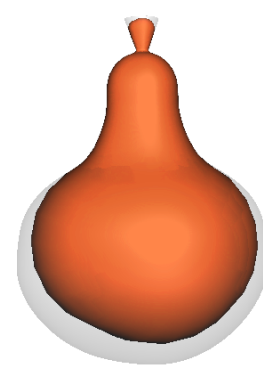

1 sheet

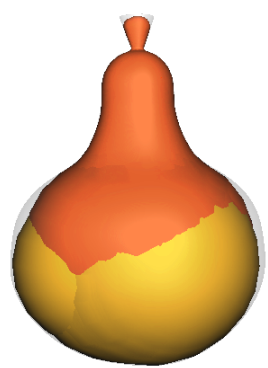

2 sheets

(b) $94.82 \%$

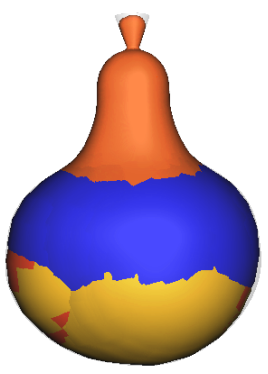

4 sheets

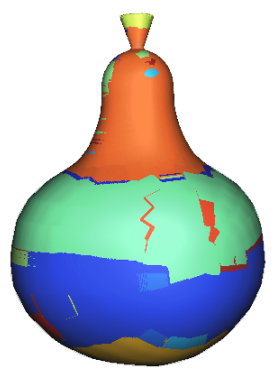

38 sheets

(d) $100 \%$

Figure 13: A pear model approximated using a progressively larger number of sheets of medial spheres. Most of the shape is covered using 4 sheets only.

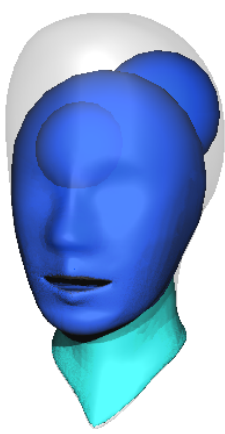

2 sheets

(a) $68.024 \%$

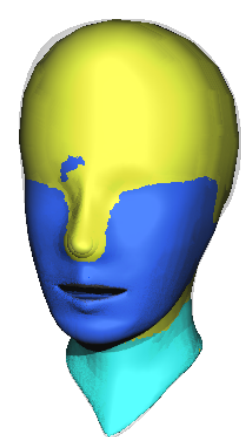

4 sheets

(b) $90.23 \%$

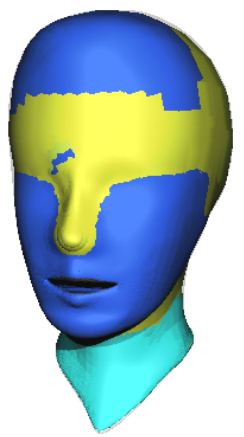

6 sheets

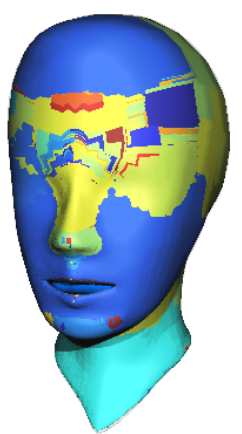

60 sheets

(d) $100 \%$

Figure 14: A head model approximated using a progressively larger number of sheets of medial spheres. Most of the shape is covered using 6 sheets only. 